\title{
Using Neural Networks To Control The Process of Plasma Etching and Deposition
}

\author{
G. Erten, A. Gharbi ${ }^{*}$, F. Salam ${ }^{*+}$, T. Grotjohn ${ }^{+}$, J. Asmussen $^{+}$ \\ Innovative Computing Technologies, Inc., Okemos, Michigan \\ * Circuit, Systems and Artificial Neural Networks Laboratory \\ ${ }^{+}$Electronic Surface Properties of Materials (ESPM) Center \\ Michigan State University, East Lansing, MI 48824 \\ ictech@mcimail.com, salam@ee.msu.edu
}

\begin{abstract}
Neural architectures are proposed to model and control plasma etching and deposition processes in semiconductor wafer manufacturing. Static and dynamic neural networks are used to develop plant models and inverse models. A single-hidden layer feedforward neural network model learns to identify the system's input-output relationship. Another single-hidden layer feedforward neural controller learns to model the inverse relationship of the plant. The trained controller, in series with appropriate filters, is then used to control the plasma machine in etching and deposition processes. The paper demonstrates how neural networks can learn both the modeling and control tasks in this nonlinear and complex process.
\end{abstract}

\section{INTRODUCTION}

Chemically reactive plasma discharges are widely used to modify the surface properties of materials. Plasma processing technology is vitally important to several of the global manufacturing industries in the world, including semiconductors, automotive, steel, biomedical and toxic waste management industries [1]. Specifically, plasma-based surface processes are indispensable for wafer manufacturing of microelectronic integrated circuits and micro-electromechanical systems (MEMS). A series of plasma processing steps, namely, deposit or grow, dope or modify, etch or remove, are repeated frequently and alternately in the manufacturing of modern micro-electronic integrated circuits and systems.

As the feature size of technology continues to decrease below the present day 0.35 micron, accurate and repeatable processing becomes essential. Moreover, due primarily to economic pressures, the shift in the micro-electronic manufacturing industry is towards the processing of a single but large wafers in compact adjustable machines known as clustor tools. Such desired precision and repeatability can only be achieved by the application of advanced automated methodologies of identification and control.

The analysis and design techniques of identification and control are well-systemized and articulated for linear systems. However, such techniques are only beginning to be formulated for nonlinear or time-varying systems. Moreover, the manufacturing processes do not in reality lend themselves to closed form tractable models of maps, differential or discrete equations. Indeed, such a process most often possesses nonlinearities, hysteresis, time-variations and temperature dependencies which are not well-characterized. Under these conditions, it becomes imperative that approaches which combine the accuracy and soundness of system analysis with novel computing methods, such as neural networks and/or fuzzy logic, be used to tackle the identification and control needs without necessarily deriving explicit basic-principles mathematical models. In this context, supported by analytical foundations, neural networks and fuzzy logic techniques may serve as an alternate or complementary tool. Such techniques derive their information from measured data and aspire to improve their modeling as more data become available. Hence learning on-line can be achieved by these multi-inputmulti-output, nonlinear form of adaptive systems.

Different approaches in modeling semiconductor fabrication processes have been developed previously including statistical experimental design, response surface methodology (RSM) and statistical process control [2]. Few authors, however, have investigated modeling reactive ion etching processes via neural networks [3-6]. 
This paper is organized as follows. In section 2, a brief description of the plasma processing system used for the experiments is given. In section 3, the static and dynamic neural architecture will be considered for modeling. In section 4, the control problem is defined and formulated. In section 5, computer simulation results are presented to show the performance of the developed controller on the data-based plasma machine model. Finally, in section 6 we summarize our conclusions and assessment of the methodology in modeling and controlling complex plasma systems.

\section{SYSTEM DESCRIPTION}

The plasma processing machine used for the experiments consists [7] of a microwave plasma disc reactor (MPDR) incorporated into vacuum, gas handling and microwave subsystems. The MPDR consists of a microwave cavity, the discharge chamber and the electron cyclotron resonance (ECR) baseplate. The MPDR utilizes a single mode electromagnetic cavity. The electric field distribution within the discharge can be controlled by selecting the appropriate resonant mode of the cavity such that the microwave energy is focused onto the discharge chamber. In the discharge chamber, the flowing gas creates the plasma which under most operating conditions has an electron density between $10^{10} \mathrm{~cm}^{-3}$ and $10^{12} \mathrm{~cm}^{-3}$. Therefore the microwave radiation inside the cavity cannot propagate through the plasma into the processing chamber. The baseplate supplies the working gases into the ECR magnetic field within the discharge region. In Fig. 1, we have labeled each component of the microwave cavity and the overall system in consecutive numerals to facilitate the system description.

\section{THIS FIGURE IS ATTATCHED TO THE DOCUMENT}

Fig. 1: The Plasma Processing Machine

A tunable microwave cavity (9) is used to match impedances between the plasma and the microwave power source. The body of the cavity consists of a brass cylinder. The upper end of the cylinder is terminated by a movable plate or sliding short (8). In the lower end of this resonant structure resides the discharge chamber (2) and the base plate (12). Microwaves are introduced into this volume via a short antenna (7) which emerges from the center conductor of a 7/8" diameter, rigid coaxial transmission line (6). The length of the antenna, inside the cavity is adjustable to allow impedance matching between the microwave power source and the cavity. The height of the cavity may be varied by moving the sliding short. Using this two degrees of freedom it is possible to match the impedance of the cavity/plasma system to the $50 \mathrm{~W}$ microwave feed.

Besides the ECR magnets, the baseplate consists of the discharge chamber (2), working gas inlets (3), and water cooling. The plasma is contained in the central region of the baseplate under a fused quartz window (1) which is vacuum sealed to the baseplate by a silicone gasket. The dielectric window is held in place by the pressure differential between the evacuated plasma region and the atmospheric pressure of the microwave cavity region.

Although plasma processing may be accomplished in the discharge chamber, the region below this region will be referred to as processing chamber (4). This region has the advantage of being free from the microwave electromagnetic fields and static magnetic fields which permeate the discharge chamber. The processing chamber is simply a pyrex cylinder which is capped by a stainless steel plate with a seven inch diameter vacuum port for the MPDR. The downstream end of the chamber is also terminated by a stainless steel plate which hosts a 10 inch diameter inlet (15) to the diffusion pump. 
Situated in the processing chamber is a two inch diameter aluminum substrate holder (14) which can be electrically biased and manually positioned, so that a substrate (13) can be positioned ideally into the down streaming plasma.

Through digital and A/D ports, the microwave incident and reflected power are read into a computer. The computer performs the subtraction of reflected power from the incident power to obtain the absorbed power in the plasma. The cavity height, measured by a sensor, is also being read by the computer as well as the actual value of the pressure, provided by the pressure controller. The computer also acquires the plasma system output including the ion density measured via probe (5), located under the discharge chamber, and the strength of the electric field in the cavity (10) measured also with a probe. Table 1 lists the inputs and outputs considered in this work.

\begin{tabular}{|l|l|l|l|}
\hline \multicolumn{2}{|c|}{ Inputs } & \multicolumn{2}{c|}{ Outputs } \\
\hline Parameter & $\begin{array}{c}\text { Var } \\
{[\text { Units] }}\end{array}$ & \multicolumn{1}{c|}{ Parameter } & \multicolumn{1}{|c|}{ Var [Units] } \\
\hline Incident Power & $\mathrm{P}_{\text {inc }}[\mathrm{W}]$ & Absorbed Power & $\mathrm{P}_{\mathrm{abs}}[\mathrm{W}]$ \\
\hline Pressure & $\mathrm{P}[\mathrm{mTorr}]$ & Number of Ions & $\mathrm{N}_{\mathrm{i}}\left[10^{11} \mathrm{~cm}^{-3}\right]$ \\
\hline Cavity Size & $\mathrm{L}_{\mathrm{s}}[\mathrm{mm}]$ & Electric Field & $\mathrm{Pc}-16[\mathrm{v} / \mathrm{m}]$ \\
\hline
\end{tabular}

Table 1: Inputs and outputs of the plasma system

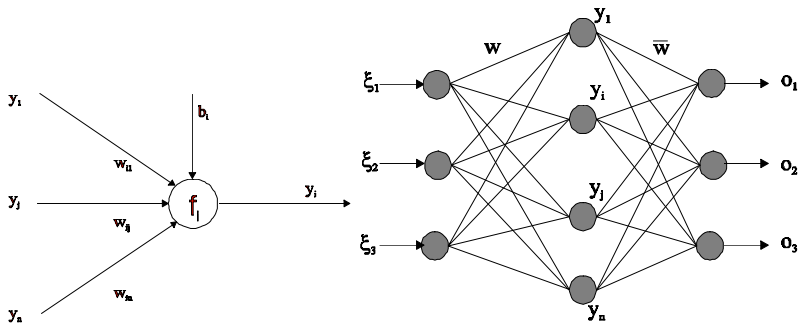

Fig. 2: Feedforward Neural Networks

\section{NEURAL NETWORKS}

Neural networks [8,9] can be used to design models that represent input/output relationships. The main building component of a neural network is the neuron. Each neuron has a number of parameters called weights and biases. The interactions of the individual neurons and the change of the values of these parameters are what defines the functionality of the whole network. (See Fig. 2.) The goal is then to choose the weights and biases of the network to achieve the desired static input/output relationship. Depending on the nature of the data, static or dynamic neural network architectures may be considered.

\subsection{Static Neural Network}

The activity of each neuron is defined by:

$$
o_{i}=f_{i}\left(\sum_{j=1}^{n} w_{i j} y_{j}+b_{i}\right)
$$

where $\mathrm{o}_{j}$ is the $j^{\text {th }}$ neuron's output, $b_{j}$ is the $j^{\text {th }}$ neuron's bias, $w_{i j}$ is the weight connecting the $j^{\text {th }}$ neuron's output to the $i^{\text {th }}$ neuron's input and $f_{i}$ is the $\mathrm{i}^{\text {th }}$ neuron's activation function.

A neural network is constructed by connecting a set of neurons to each other. Most commonly used architecture to design such neural networks is the multilayer feedforward architecture where the neurons are lined up in columns (or layers) and every neuron within a layer is connected only to the neurons from the previous layers.

The parameters (the weights and biases) are then updated using the gradient descent method $[8,9]$ :

$$
\begin{array}{ll}
\Delta \bar{w}_{i j}=\eta \sum_{\mu} \bar{\delta}_{i}^{\mu} y_{j}^{\mu} \text { and } \Delta \bar{b}_{i}=\eta \sum_{\mu} \bar{\delta}_{i}^{\mu} \quad \text { where } \bar{\delta}_{i}^{\mu}=\left(o_{i}^{\mu}-\zeta_{i}^{\mu}\right) f^{\prime}\left(\bar{h}_{i}^{\mu}\right) \\
\Delta w_{i j}=\eta \sum_{\mu} \delta_{i}^{\mu} \xi_{j} \text { and } \Delta b_{i}=\eta \sum_{\mu} \delta_{i}^{\mu} & \text { where } \delta_{i}^{\mu}=f^{\prime}\left(h_{i}^{\mu}\right) \sum_{k} \bar{\delta}_{k}^{\mu} \bar{w}_{k i}
\end{array}
$$

and $\eta$ is the learning rate. The above learning rule is commonly known in the literature as the standard back-propagation learning. The back-propagation learning algorithm is based on the (discrete) gradient descent method which can be very slow if $\eta$ is small and may oscillate or diverge if $\eta$ is too large. Possible ways of dealing with this problem are to use adaptive learning rates and the inclusion of the momentum term $[7,8]$. Other more sophisticated techniques include the use of more appropriate energy functions, new structures, and/or novel learning rules. 


\subsection{Dynamic Neural Networks}

A recurrent (dynamic) neural network is capable of dealing with time-varying input and/or output through their own temporal dynamics. In this case, we model the activity of each neuron $y_{i}$ by the following dynamic equation:

$$
\theta(\dot{y}, y, T)=\varphi(W, K, B, y, \xi), \quad y\left(t_{0}\right)=y_{0}
$$

where $\theta(\dot{y}, y, T)=\left\{\begin{array}{cc}T \dot{y}+y & M 1 \\ \dot{y}+T y & M 2 \\ T^{2} \dot{y}+y & M 3\end{array} \quad\right.$ and $\quad \varphi(W, K, B, y, \xi)=\left\{\begin{array}{cc}f(W y)+K \xi & \text { Architecture 1 } \\ W f(y)+K \xi & \text { Architecture 2 } \\ f(W y+K \xi) & \text { Architecture 3 } \\ W f(y)+K g(\xi) & \text { Architecture 4 }\end{array}\right.$

For example, a neuron of M1 and architecture 1 is represented by:

$$
\tau_{i} \dot{y}_{i}=-y_{i}+f\left(\sum w_{i j} y_{j}\right)+\sum k_{i m} \xi_{m}, \quad y_{i}\left(t_{0}\right)=y_{i 0}
$$

where $\tau_{\mathrm{i}}$ is the time constant of $\mathrm{i}^{\text {th }}$ neuron, $\mathrm{y}_{i}$ is the activity of $\mathrm{i}^{\text {th }}$ neuron, $\mathrm{f}_{i}$ is an activation function of the $\mathrm{i}^{\text {th }}$ neuron, $w_{i j}$ is the weight of the $\mathrm{j}^{\text {th }}$ neuron $\mathrm{y}_{j}$ to the $\mathrm{i}^{\text {th }}$ neuron $\mathrm{y}_{i}$ and $k_{i m}$ is the weight of the $\mathrm{m}^{\text {th }}$ input $\xi_{\mathrm{m}}$ to the $\mathrm{i}^{\text {th }}$ neuron $\mathrm{y}_{i}$

In recurrent neural networks, any neuron in the network can be connected to any other neuron in the net. The user has to define the input, output and hidden neurons, and the connections among them. For example, an output neuron may be connected to an input. Nevertheless, each neuron in the network should belong to at least one of the three sets of neurons. $N_{i}$ : set of input neurons, $N_{h}$ : set of hidden neurons, $N_{o}$ : set of output neurons. The network's connectivity and structure need to accommodate the potentially complex (time-varying) sets of input/output training data.

The objective of the training is to minimize an error functional between the observed output of the neural network $\mathrm{y}(t)$ and the desired (target) waveform $\zeta(t)$ :

$$
E[W, K, T]=\frac{1}{2} \int_{t_{0}}^{t_{1}} \sum_{k \in N_{o}}\left(y_{k}(t)-\zeta_{k}(t)\right)^{2} d t
$$

subject to the constraint of the activities of the neurons $\mathrm{y}(t)$ as defined above. Thus, we define the augmented performance index:

$$
J[W, K, T]=\int_{t_{0}}^{t_{1}} \mathcal{L}(\dot{y}, y, \lambda, W, K, T, t) d t
$$

where the Langrange function is defined by:

$$
\mathcal{L}=\sum_{m=1}^{n} \delta_{m m}^{m \in N_{o}}\left(y_{m}-\zeta_{m}\right)^{2}+\lambda_{m}\left(\theta_{m}-\varphi_{m}\right)
$$

From the calculus of variation and optimization principles, the integral leads to the adjoint system:

$$
\frac{d}{d t}\left\{\left(\frac{\partial \theta}{\partial \dot{y}}\right)^{T} \lambda\right\}=\left(\frac{\partial(\theta-\varphi)}{\partial y}\right)^{T} \lambda+\Delta^{o}(y-\zeta), \quad \lambda\left(t_{1}\right)=0 .
$$

The network (matrix) parameters $W, K$ and T are updated using the gradient descent laws:

$$
\Delta p=-\eta \frac{\partial J}{\partial p}=-\eta \int_{i_{0}}^{t_{0}} \frac{\partial \mathcal{L}}{\partial p} d t, \quad p=W, K, T .
$$

By computing the partial derivatives, one obtains:

$$
\Delta W=\eta \int_{t_{0}}^{t_{1}} \lambda\left(\frac{\partial \varphi}{\partial W}\right)^{T} d t, \quad \Delta K=\eta \int_{t_{0}}^{t_{1}} \lambda\left(\frac{\partial \varphi}{\partial K}\right)^{T} d t \quad \text { and } \Delta T=-\eta \int_{t_{0}}^{t_{1}} \lambda\left(\frac{\partial \theta}{\partial T}\right)^{T} d t
$$

We now describe our algorithm/procedure to run simulations of recurrent neural networks as follows:

Step 1: Choose initial (matrix) parameters $W_{0}, K_{0}, \mathrm{~T}_{0}$

Step 2: Integrate forward the state $y(t)$

Step 3: Integrate Backward the state $\lambda(t)$

Step 4: Update the parameters using the above update equations

Step 5: If $y(t) \stackrel{\text { does not converge to }}{\longrightarrow} \zeta(t)$, go to step 2 . 


\section{The CONTROL FORMULATION AND STRUCTURE}

In the context of this paper, the plasma etching and deposition processing and control is to provide the correct incident power, pressure and cavity size inputs to drive the plant to the desired absorbed power, number of ions and electric field outputs. Traditional nonlinear control theory requires a mathematical model of the plant to be controlled. It may suggest control procedures or linearize the plant around a number of operating points. However, in addition to requiring a mathematical model of the plant, such an approach is usually computationally intensive, requires considerable design effort, and does not apply to poorly modeled complex nonlinear systems.

The objective is then to train a neural network controller to produce the correct input to drive the plant to the desired output. A number of different approaches for training a controller have been described in the literature [10-12] such as the reinforcement learning. In this work, we use the generic control structure shown in fig. 3 below.

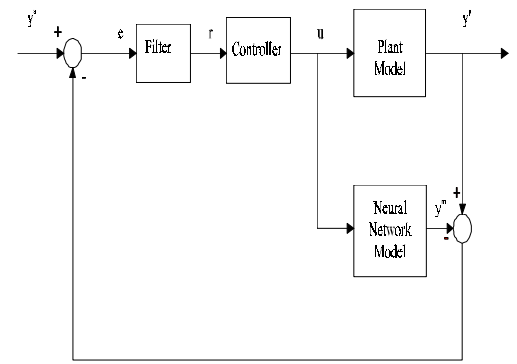

(a) Overall Neural Control Structure

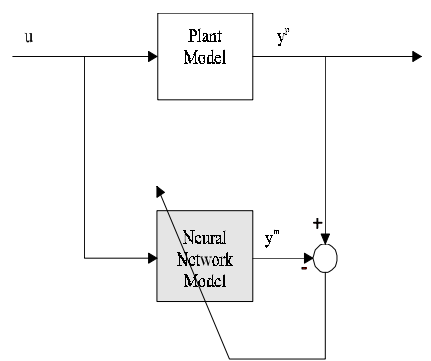

(b) Plant Modeling Structure

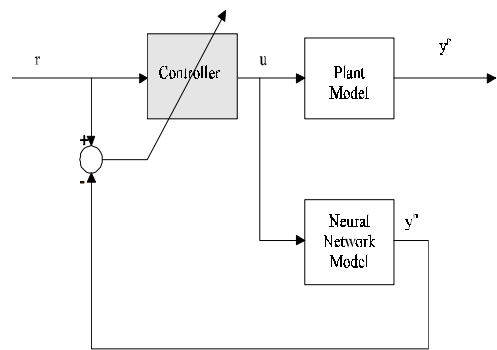

(c) Controller Modeling Structure

Fig. 3. Structures

In [11], the authors describe a control structure such as the one shown in Fig. 3(a) to control a nonlinear plant P. They proposed a three step-procedure to carry out this task: (i) design a neural network model, $\mathrm{M}$, that represents the plant, (ii) design a neural network model, $\mathrm{C}$, that represents the inverse functionality of the plant, and (iii) use the structure in Fig. 3(a) with an appropriate choice of the filter, F, to control the plant.

With the structures as defined in Fig 3(b,c), both a model and a controller neural networks were developed. The plant modeling was discussed in the previous section. The best model of the plant obtained was considered as the mathematical replica of the plant $\mathrm{P}$. The second best model of the input/output relationship was chosen as the neural network model $\mathrm{M}$ itself. Simulations of the performance of the inverse model (controller) is shown in Fig. 4 below. Note that the physical data was scaled to within the range $[-1,1]$ to enable convenient processing.

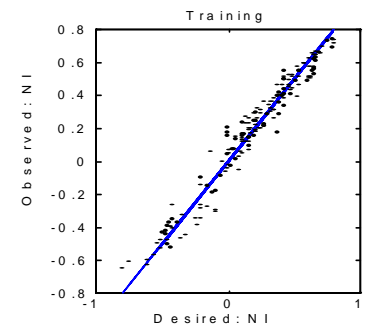

(a) Neural Modeling
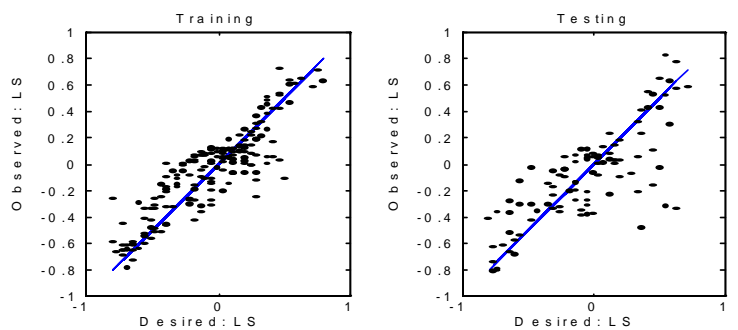

(b) Neural Inverse Modeling

Fig. 4: Neural Networks Performance

\section{SIMULATIONS}

A filter $\mathrm{F}$ to achieve the control of the plant using the structure described in Fig. 3(a) was designed. A second order filter to adjust the gain in series with an integrator to minimize the backpropagation error were used. The filter is of the form:

$$
F(s)=K_{p} \frac{w_{n}^{2}}{s^{2}+2 \zeta w_{n} s+w_{n}^{2}}+\frac{K_{e}}{s}
$$

With an appropriate choice of parameters, the output of the plant will match some desired reference that is set by the user. By considering $K_{p}=1.1, \quad w_{n}=10, \zeta=0.7$ and $K_{e}=1000$, the closed-loop system is able to track the set reference. Figures 5 and 6 represent the performance of the controller for a sample of the trained data. It can be observed that the 
plant reaches the desired target within $10 \mathrm{msec}$ in simulation with nominal step size of $0.5 \mathrm{msec}$. In this experiment, it is desired to produce the necessary control signal vector $u$ that will drive the plant to the following reference $(-0.6757$, $0.1636,-0.7947)$. This data corresponds to an absorbed power of $70.3974 \mathrm{~W}$, a number of ions of $2.363 \times 10^{11}$ and an electric field of magnitude $0.0429 \mathrm{~V} / \mathrm{m}$. As it is observed in Figures 5 and 6, the plant has tracked these reference signals within $10 \mathrm{msec}$. A control signal of $(0.5052,-9292,0.9389)$ was necessary to accomplish this task. When these values are converted to their physical domain, they correspond to $(81.9180,2.2173,296.1167)$. This pattern is not included in the trained or tested data set. This shows that the designed control has the potential to generalize to other input/output pairs that are not included in the collected data set.
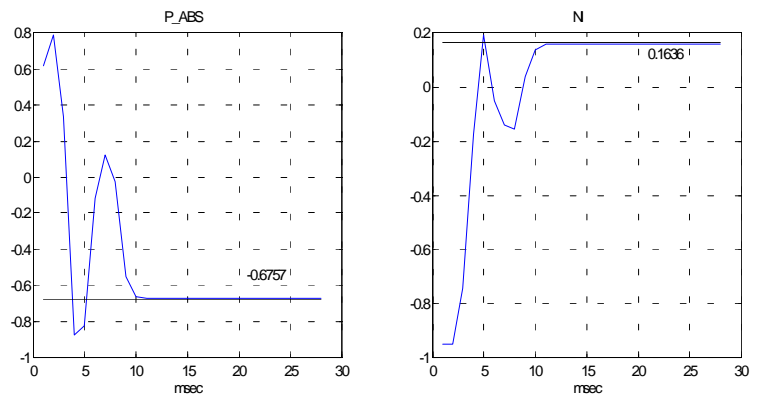

Fig. 5: Output Tracking
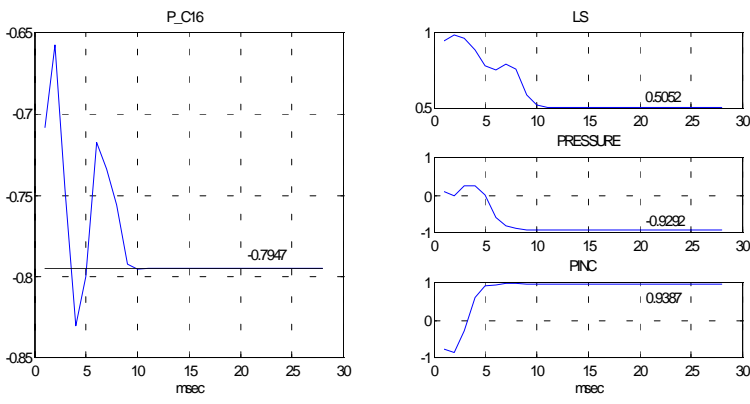

Fig. 6: Control signals

\section{CONCLUSION}

The paper addresses a problem of controlling the processing of plasma etching and deposition process utilizing neural networks to achieve a reconfigurable nonlinear control design. The methodology shows promise and superior performance to control the processing of plasma etching and deposition given that reliable analytical control theory techniques do not exist for controlling such complex nonlinear process.

\section{ACKNOWLEDGMENT}

This work was supported in part by the NSF under SBIR grant DMI-9461031, IC Tech, Inc., and the Michigan Research Excellence Fund (REF).

\section{REFERENCES}

[1] Allan J. Lichtenberg, Michael A. Liebmann, "Principles of Plasma Discharges and Materials Processing", John Wiley \& Sons Inc., 1994.

[2] C. J. Spanos, "Statistical Process Control in Semiconductor Manufacturing", Proceedings of the IEEE, Vol. 80, No. 6, pp. 819-30, June 1992.

[3] Gary S. May, "Manufacturing IC's the Neural way", IEEE Spectrum, pp. 47 - 51, September 1994.

[4] F. Nadi, A. Agogino, D. Hodges, "Use of influence diagrams and neural networks in modeling semiconductor manufacturing processes", IEEE Transactions on Semiconductor Manufacturing, Vol. 4, No.1, February 1991.

[5] Byunghwan Kim, Gary S. May, "An optimal model for plasma etching", IEEE Transactions on Semiconductor Manufacturing, Vol. 7, No.1, February 1994.

[6] Michael D. Baker, Christopher D. Himmel, Gary S. May, "Time series modeling of reactive ion etching using neural networks", IEEE Transactions on Semiconductor Manufacturing, Vol. 8, No.1, February 1995

[7] J. Hopwood, D. K. Reinhard and J. Asmussen, Journal Vacuum Science Technology, A 8, pp. 3103, 1990.

[8] N. Bose and P.Ling, "Neural Network Fundamentals with Graphs, Algorithms and Applications, McGraw-Hill, 1996

[9] John Herz, Andreas Krogh and Richard Palmer, "Introduction to the Theory of Neural Computation", AddisonWesley, 1991.

[10] K. Narendra and K. Parthsarathy, "Identification and Control of Dynamical Systems Using Neural Networks", IEEE Transactions on Neural Networks, vol. 1, no. 1, pp. 4-27, 1990.

[11] Widrow, N. K. Gupta and S. Maitra, "Punish/Reward: Learning with a Critic in Adaptive Threshold Systems", IEEE Transactions on Control Systems Magazine, , October 1989.

[12] Anderson, "Learning to Control an Inverted Pendulum Using Neural Networks", IEEE Transactions on Control Systems Magazine, vol. 9, no. 3, April 1989. 